\title{
The Relationship of Hepatitis Antibodies and Elevated Liver Enzymes with Impaired Fasting Glucose and Undiagnosed Diabetes
}

\author{
Arch G. Mainous, III, PhD, Vanessa A. Diaz, MD, MS, Dana E. King, MD, MS, \\ Charles J. Everett, MD, MS, and Marty S. Player, MD, MS
}

Objective: The aim of this study was to examine the relationship of hepatitis antibodies and liver enzymes with impaired fasting glucose and undiagnosed diabetes in adults.

Methods: We analyzed the National Health and Nutrition Examination Survey, 1999 to 2004, a nationally representative sample of the noninstitutionalized US population. Among adults (aged $\geq 20$ years of age) who were not problem drinkers, we examined hepatitis $B$ and $C$ antibodies and the liver enzymes aspartate aminotransferase (AST), alanine aminotransferase (ALT), and $\gamma$ glutamyl transaminase (GGT) with impaired fasting glucose and undiagnosed diabetes (unweighted, $n=5234$; weighted, $n=$ $172,626,805)$. Logistic regression models were computed controlling for major risk factors that drive diabetes screening, including age, gender, race, diagnosed hypertension, diagnosed hypercholesterolemia, and obesity.

Results: In unadjusted analyses $51 \%$ of individuals with undiagnosed diabetes have elevated GGT versus $20 \%$ of individuals without diabetes or impaired fasting glucose $(P=.01)$. Similarly, $43 \%$ of individuals with undiagnosed diabetes have elevated ALT versus $23 \%$ of individuals without diabetes or impaired fasting glucose $(P=.01)$. AST and Hepatitis $C$ antibodies were not associated with undiagnosed diabetes. In adjusted analyses, elevated GGT (odds ratio, 2.15; 95\% CI, 1.44-3.20) and ALT (odds ratio, 1.84; 95\% CI, 1.06-3.20) are associated with undiagnosed diabetes. Similarly, in adjusted analyses, elevated GGT (odds ratio, 1.23; 95\% CI, 1.00-1.53) and ALT (odds ratio, 1.44; 95\% CI, 1.151.79) are associated with impaired fasting glucose. Hepatitis antibodies, reporting a current liver problem, or AST were associated with having undiagnosed diabetes in adjusted analyses.

Conclusions: Liver function is associated with undiagnosed diabetes and impaired fasting glucose and may justify further investigation as a risk stratification variable for undiagnosed diabetes or impaired fasting glucose. (J Am Board Fam Med 2008;21:497-503.)

Insulin resistance can be thought of as being composed of hepatic and extrahepatic components that play a role in impaired fasting glucose. ${ }^{1}$ Conse-

This article was externally peer reviewed.

Submitted 21 February 2008; revised 20 May 2008; accepted 21 May 2008.

From the Department of Family Medicine, Medical University of South Carolina, Charleston.

Funding: This project was supported in part by grant 1 P30AG021677 from the National Institute on Aging and by grants from the Robert Wood Johnson Foundation.

Conflict of interest: none declared.

Corresponding author: Arch G. Mainous III, PhD, Department of Family Medicine, Medical University of South Carolina, 295 Calhoun St., Charleston, SC 29425 (E-mail: mainouag@musc.edu).

\footnotetext{
See Related Commentary on Page 487.
}

quently, hepatic problems affect insulin resistance. Hepatitis is associated with insulin resistance. ${ }^{2}$ Hepatitis $\mathrm{C}$ virus (HCV) is associated with insulin resistance, and clearance of $\mathrm{HCV}$ improves insulin resistance. ${ }^{3}$ This evidence would suggest that hepatitis and impaired liver function may be associated with the development of diabetes.

Some limited data on the relationship between liver enzymes and diabetes has suggested that elevated liver enzymes, aspartate aminotransferase (AST), alanine aminotransferase (ALT), and yglutamyl transaminase (GGT) are associated with the development of diabetes. ${ }^{4-8}$ Other data has suggested that the relationship between liver enzymes and diabetes may be modified by the presence of inflammation, with C-reactive protein (CRP) playing a predictive role with liver enzymes and diabetes. ${ }^{9}$

Because of the asymptomatic nature of type 2 diabetes, between one third and a half of individuals 
with diabetes are undiagnosed. ${ }^{10}$ The prevalence of undiagnosed diabetes for adults in the United States has been estimated at $2.4 \%$, representing 4.9 million adults and nearly $30 \%$ of all diabetes in the United States in 1999 to $2000 .{ }^{11}$ A significant proportion of people $(25 \%)$ with undiagnosed diabetes have signs of nephropathy, suggesting that undiagnosed diabetes is not a benign condition. ${ }^{12}$ Consequently, different risk factors for undiagnosed diabetes have been identified as ways to guide screening strategies. ${ }^{13-16}$ The American Diabetes Association recommends screening men and women $\geq 45$ years of age for undiagnosed type 2 diabetes. ${ }^{17}$ The US Preventive Services Task Force recommends screening for diabetes among adults if they have the risk factor of hypertension. ${ }^{18}$ Diabetes UK recommends screening of white people $>40$ years of age and people from black, Asian, and minority ethnic groups $>25$ years of age with one or more major risk factors. ${ }^{19}$

There is little evidence as to whether the presence of hepatitis antibodies and elevated liver enzymes is associated with either current impaired fasting glucose or undiagnosed diabetes. Thus, the purpose of this study was to examine the relationship of hepatitis antibodies and liver enzymes with impaired fasting glucose and undiagnosed diabetes. We hypothesized that elevated liver enzymes will be associated with the presence of impaired fasting glucose and undiagnosed diabetes.

\section{Methods}

The data for this study were derived from the National Health and Nutrition Examination Survey (NHANES) 1999 to 2004, a nationally representative sample of the noninstitutionalized US population. The NHANES design includes an oversampling of minorities and an ability to make population estimates. More information on the methodology of the NHANES 1999 to 2004, including laboratory assessment, can be found at the National Center for Health Statistics (NCHS) website. ${ }^{20}$ This study included fasting participants $\geq 20$ years old who were not problem drinkers of alcohol. Problem drinking was defined as $>14$ drinks per week for men or $>7$ drinks per week for women. We excluded binge drinkers, defined as $\geq 5$ drinks during 1 day per week. ${ }^{21}$

\section{Variables}

Diabetes and Impaired Fasting Glucose

Diagnosed diabetes was defined as self-report of a doctor's diagnosis of diabetes. Undiagnosed diabetes was defined as patients reporting that they had not been diagnosed by a doctor with diabetes but who had fasting plasma glucose of $\geq 126 \mathrm{mg} / \mathrm{dL}$. Impaired fasting glucose was defined as patients not diagnosed with diabetes who had fasting plasma glucose of 100 to $125 \mathrm{mg} / \mathrm{dL}$. Those not diagnosed with diabetes who had fasting plasma glucose $<100$ $\mathrm{mg} / \mathrm{dL}$ were classified as normal. ${ }^{16}$

\section{Hepatitis Antibodies and Liver Function}

Hepatitis B core antibody and hepatitis C antibody (confirmed) were classified as positive or negative. The Ortho HBc Enzyme-Linked Immunosorbent Assay (ELISA) Test System is a qualitative ELISA for the detection of total antibody to anti- $\mathrm{HBc}$ in human serum or plasma. The Chiron RIBA 3.0 Strip Immunoblot Assay (SIA; Chiron Corporation, Inc., Emeryville, CA) is an in vitro qualitative enzyme immunoassay for the detection of antibody to hepatitis $\mathrm{C}$ virus in human serum or plasma. The SIA was used to confirm hepatitis C identified using an anti-hepatitis $\mathrm{C}$ virus screening ELISA. CRP was classified as low $(<0.3 \mathrm{mg} / \mathrm{dL})$ or elevated $(\geq 0.3 \mathrm{mg} / \mathrm{dL}){ }^{22}$ After previous research relating liver enzymes to the development of diabetes, liver enzymes were classified as low (below the fourth quartile) or high (fourth quartile). ALT $\geq 27.78$ $\mathrm{u} / \mathrm{L}$ was classified as elevated; AST $\geq 25.51 \mathrm{u} / \mathrm{L}$ was classified as elevated; and GGT $\geq 29.48 \mathrm{u} / \mathrm{L}$ was classified as elevated. ${ }^{4-6} \mathrm{We}$ also evaluated patients based on their answers to the questions, Has a doctor or health professional ever told you that you have a liver condition? and Do you still have a liver condition?

\section{Control Variables}

In addition to age and gender, the control variable of self-reported race/ethnicity was created out of a question about the person's race and an additional question about Hispanic ethnicity. Race/ethnicity was classified as non-Hispanic white, non-Hispanic black, Hispanic, and other. Body mass index was calculated as $\mathrm{kg} / \mathrm{m}^{2}$ from height and weight measured during the NHANES physical examination and was analyzed as a continuous variable. Because hypercholesterolemia and hypertension are risk factors that drive screening for diabetes we felt that 
it was important to control for previously diagnosed hypercholesterolemia and hypertension. Previous diagnosis would allow health care providers to be aware of the condition. High cholesterol was defined as the self-report of a doctor having told the participant he or she had high cholesterol. Those who had not been tested were considered to have not been diagnosed with high cholesterol. Hypertension was defined as the self-report of a doctor ever having told the participant he or she had high blood pressure or hypertension.

\section{Analysis}

Because this survey is based on a complex sampling design that makes it representative of the noninstitutionalized US population, we are able to make nationally representative estimates. We used SUDAAN software (Research Triangle Institute, Research Triangle Park, NC) to account for the weighting and complex sampling design.

We evaluated bivariate relationships between hepatitis antibodies, CRP, and liver enzymes with diagnosed diabetes, undiagnosed diabetes, impaired fasting glucose, and normal participants using $\chi^{2}$ analysis. Because biomarkers may be affected by treatment regimens once an individual has been diagnosed with diabetes, we conducted additional analyses focusing on individuals with undiagnosed disease. We conducted adjusted logistic regressions, excluding patients with diagnosed diabetes and predicting undiagnosed impaired fasting glucose and undiagnosed diabetes alone. Control variables included age, gender, race/ethnicity, body mass index, previous diagnosis of hypercholesterolemia, and previous diagnosis of hypertension.

Finally, we examined whether the association between liver enzymes and impaired fasting glucose or undiagnosed diabetes was affected by elevated CRP. We created variables with 4 categories: 1) elevated liver enzyme, elevated CRP; 2) low liver enzyme, elevated CRP; 3 ) elevated liver enzyme, low CRP; and 4) low liver enzyme, low CRP. The combination of GGT, AST, and ATL with CRP were used in 3 separate logistic regressions to predict undiagnosed diabetes or impaired fasting glucose, adjusted for the previously identified control variables. In the analyses of this study we assumed a level of $P<.05$ to represent statistical significance.
Table 1. Characteristics of the Sample from the National Health and Nutrition Examination Survey 1999 to 2004

Unweighted (n)

5234

Weighted (n)

Diabetes (\%)

Diagnosed diabetes $\quad 7.0$

Undiagnosed diabetes $\quad 2.6$

Impaired fasting glucose $\quad 25.5$

Normal

64.9

Hepatitis B core antibody

Positive (\%)

6.0

Hepatitis C core antibody

Positive (\%)

CRP (\%)

Low $(<0.3 \mathrm{mg} / \mathrm{dL})$

61.3

High $(\geq 0.3 \mathrm{mg} / \mathrm{dL})$

GGT (\%)

Low $(<29.48 \mathrm{u} / \mathrm{l})$

74.6

High $(\geq 29.48 \mathrm{u} / \mathrm{l})$

25.4

AST (\%)

Low $(<25.51 \mathrm{u} / \mathrm{l})$

73.2

High $(\geq 25.51 \mathrm{u} / \mathrm{l})$

26.8

$\operatorname{ALT}(\%)$

Low $(<27.78 \mathrm{u} / \mathrm{l}) \quad 73.0$

$\operatorname{High}(\geq 27.78 \mathrm{u} / \mathrm{l}) \quad 27.0$

Age (\%)

20 to 44 years $\quad 50.5$

45 to 64 years $\quad 33.3$

$\geq 65$ years $\quad 16.1$

Gender (\%)

Male

46.0

Female $\quad 54.0$

Race/Ethnicity (\%)

Non-Hispanic white $\quad 72.3$

Non-Hispanic black $\quad 10.9$

Hispanic 12.6

Other $\quad 4.1$

Body mass index $\left(\mathrm{kg} / \mathrm{m}^{2}\right)$

$<25.0$

35.0

$25.0-29.9$

33.9

$\geq 30.0$

Previously diagnosed (\%)

High cholesterol

Yes

No or not tested $\quad 72.3$

Hypertension

Yes

27.2

No

72.8

CRP, C-reactive protein; GGT, yglutamyl transferase; AST, aspartate aminotransferase; ALT, alanine aminotransferase. 
Table 2. Hepatitis B Core Antibody, Hepatitis C Antibody (Confirmed), C-Reactive Protein, and Liver Enzymes by Diabetes/Impaired Fasting Glucose Categories

\begin{tabular}{|c|c|c|c|c|c|}
\hline & Diagnosed Diabetes & Undiagnosed Diabetes & Impaired Fasting Glucose & Normal & $P$ \\
\hline \multicolumn{6}{|c|}{ Hepatitis B core antibody } \\
\hline Positive (\%) & 13.83 & 7.98 & 5.59 & 5.22 & .001 \\
\hline \multicolumn{6}{|c|}{ Hepatitis C antibody (confirmed) } \\
\hline Positive (\%) & 2.11 & 2.12 & 1.38 & 1.40 & .90 \\
\hline \multicolumn{6}{|l|}{ CRP (\%) } \\
\hline Low & 50.34 & 47.18 & 56.79 & 64.85 & $<.01$ \\
\hline High & 49.66 & 52.82 & 43.21 & 35.15 & \\
\hline \multicolumn{6}{|l|}{$\operatorname{ALT}(\%)$} \\
\hline Low & 70.48 & 56.98 & 65.60 & 76.88 & $<.01$ \\
\hline High & 29.52 & 43.02 & 34.40 & 23.12 & \\
\hline \multicolumn{6}{|l|}{ AST (\%) } \\
\hline Low & 73.78 & 71.67 & 67.87 & 75.22 & $<.01$ \\
\hline High & 26.22 & 28.33 & 32.13 & 24.78 & \\
\hline \multicolumn{6}{|l|}{ GGT (\%) } \\
\hline Low & 58.93 & 48.94 & 67.90 & 79.89 & $<.01$ \\
\hline High & 41.07 & 51.06 & 32.10 & 20.11 & \\
\hline
\end{tabular}

CRP, C-reactive protein; ALT, alanine aminotransferase; AST, aspartate aminotransferase; GGT, yglutamyl transferase.

\section{Results}

The characteristics of the population are presented in Table 1. A substantial proportion of the sample had elevated liver enzymes and a much smaller proportion, as might be expected, had positive antibodies for hepatitis B or C. Table 2 shows that, when liver enzymes are crossed with diabetes sta- tus, an increased proportion of individuals with undiagnosed diabetes have elevated GGT and ALT.

In adjusted logistic regressions, hepatitis antibodies are not significantly associated with either undiagnosed diabetes or the more broadly defined category including undiagnosed diabetes and the

Table 3. Adjusted Regressions Predicting Undiagnosed Diabetes or Impaired Fasting Glucose or Undiagnosed Diabetes Alone Using Hepatitis B Core Antibody, Hepatitis C Antibody (Confirmed), and Liver Enzymes*

\begin{tabular}{|c|c|c|}
\hline & Undiagnosed Impaired Fasting Glucose & Undiagnosed Diabetes \\
\hline \multicolumn{3}{|c|}{ Hepatitis B core antibody } \\
\hline Positive & $0.98(0.64-1.52)$ & $1.42(0.63-3.24)$ \\
\hline Negative & $1.00(-)$ & $1.00(-)$ \\
\hline \multicolumn{3}{|c|}{ Hepatitis C core antibody (confirmed) } \\
\hline Positive & $1.12(0.55-2.25)$ & $2.23(0.44-11.20)$ \\
\hline Negative & $1.00(-)$ & $1.00(-)$ \\
\hline \multicolumn{3}{|l|}{$\mathrm{ALT}$} \\
\hline Low & $1.00(-)$ & $1.00(-)$ \\
\hline High & $1.44(1.15-1.79)$ & $1.84(1.06-3.20)$ \\
\hline \multicolumn{3}{|l|}{ AST } \\
\hline Low & $1.00(-)$ & $1.00(-)$ \\
\hline High & $1.12(0.95-1.34)$ & $0.78(0.44-1.37)$ \\
\hline \multicolumn{3}{|l|}{ GGT } \\
\hline Low & $1.00(-)$ & $1.00(-)$ \\
\hline High & $1.23(1.00-1.53)$ & $2.15(1.44-3.20)$ \\
\hline
\end{tabular}

*Adjusted for age, gender, race/ethnicity, body mass index, high cholesterol and hypertension. All data provided as odds ratio (95\% $\mathrm{CI})$.

ALT, alanine aminotransferase; AST, aspartate aminotransferase; GGT, yglutamyl transferase. 
Table 4. Adjusted Logistic Regressions Predicting Undiagnosed Diabetes or Impaired Fasting Glucose or Undiagnosed Diabetes Alone Using C-Reactive Protein and Liver Enzymes Combined*

\begin{tabular}{lcc}
\hline & Impaired Fasting Glucose & Undiagnosed Diabetes \\
\hline ALT and CRP & & $1.00(-)$ \\
Low ALT, low CRP & $1.00(-)$ & $0.97(0.60-1.55)$ \\
Low ALT, high CRP & $1.11(0.90-1.36)$ & $1.61(0.82-3.19)$ \\
High ALT, low CRP & $1.49(1.14-1.96)$ & $2.02(0.99-4.15)$ \\
High ALT, high CRP & $1.50(1.14-1.97)$ & $1.00(-)$ \\
AST and CRP & & $1.19(0.77-1.84)$ \\
Low AST, low CRP & $1.00(-)$ & $0.91(0.45-1.84)$ \\
Low AST, high CRP & $1.10(0.93-1.31)$ & $0.78(0.34-1.82)$ \\
High AST, low CRP & $1.16(0.93-1.44)$ & $1.00(-)$ \\
High AST, high CRP & $1.19(0.91-1.56)$ & $1.08(0.66-1.77)$ \\
GGT and CRP & $1.00(-)$ & $2.26(1.31-3.89)$ \\
Low GGT, low CRP & $1.01(0.81-1.26)$ & $2.19(1.22-3.96)$ \\
Low GGT, high CRP & $1.15(0.88-1.51)$ & \\
High GGT, low CRP & $1.34(1.07-1.68)$ & \\
High GGT, high & & \\
CRP & & \\
\hline
\end{tabular}

*Adjusted for age, gender, race/ethnicity, body mass index, high cholesterol and hypertension. All data provided as odds ratio (95\% CI).

ALT, alanine aminotransferase; CRP, C-reactive protein; AST, aspartate aminotransferase; GGT, rglutamyl transferase.

prediabetic state of impaired fasting glucose (Table 3). Elevated GGT and ALT, but not AST, are associated with undiagnosed diabetes as well as impaired fasting glucose. People who report currently having a doctor-diagnosed liver condition were not associated with undiagnosed diabetes (odds ratio, 0.98 ; 95\% CI, 0.10-9.70) or impaired fasting glucose (odds ratio, 1.02; 95\% CI, 0.43-2.40).

Table 4 presents the results of the adjusted logistic regressions that include liver enzymes combined with CRP. As with the single variable analyses, in the combined variable analysis there is no relationship between undiagnosed diabetes and AST. Neither ALT nor GGT seems to indicate an interaction between liver enzymes and CRP and the likelihood of having undiagnosed diabetes. The results for impaired fasting glucose did seem to suggest that having elevated GGT and elevated CRP increased the likelihood of having impaired fasting glucose.

\section{Discussion}

The results of this study add to our knowledge of current markers that are associated with the presence of undetected diabetes. Hepatitis B was associated with diabetes in unadjusted analyses, but when other risk factors for undiagnosed diabetes were accounted for this marker was no longer significant. However, both elevated ALT and GGT were independently associated with a significantly greater likelihood of having undiagnosed diabetes and impaired fasting glucose. Previous research has suggested that elevated ALT and GGT are associated with the development of diabetes. ${ }^{4-8}$ This suggests that not only is liver function associated with the development of diabetes but it also seems to be a risk factor for currently having undiagnosed diabetes.

The finding that AST was not significantly associated may be because, although ALT is found primarily in the liver, AST is found not only in the liver but also in cardiac muscle, skeletal muscle, kidneys, brain, pancreas, lungs, leukocytes, and erythrocytes (in decreasing order of concentration). Thus, it may not be as specific a marker of the liver injury that is associated with diabetes and impaired fasting glucose.

One implication of these findings for clinical practice is that undiagnosed diabetes should be considered as a possibility in instances of elevated ALT or GGT. A finding of elevated liver enzymes may act for clinicians as a cue to consider an investigation of the possibility of undiagnosed diabetes or impaired fasting glucose, even in the absence of other common risk factors. These results suggest 
that not only is liver function a risk factor for the development of diabetes-it is also a marker that clinicians should consider for increased suspicion of undetected diabetes.

Several limitations to this study should be noted. First, although this study used a strategy for identifying undiagnosed diabetes that has been previously used in the NHANES for population-based estimates, it is not consistent with the recommendations for a clinical diagnosis of diabetes because of the one-time assessment. ${ }^{11,12}$ Second, the NHANES provides estimates for majority of individuals in the United States but it is limited to noninstitutionalized individuals in the United States, and thus the relationship between liver enzymes and undiagnosed diabetes may not hold for prisoners or other institutionalized individuals. Several variables are based on self-reports by the patients (eg, previous diagnosis by a doctor of diabetes, high cholesterol, or hypertension). Although the National Center for Health Statistics has for many years uses a cognitive laboratory to evaluate and validate items regarding receipt of health services and health behaviors, there is a possibility of response bias in these questions. ${ }^{23}$

The results presented here suggest that elevated liver tests, especially of ALT and GGT, indicate that clinicians might want to investigate whether or not a patient has undiagnosed diabetes. Liver function as a risk stratification variable for undetected diabetes or impaired fasting glucose may be worth further investigation.

\section{References}

1. Bock G, Chittilapilly E, Basu R, et al. Contribution of hepatic and extrahepatic insulin resistance to the pathogenesis of impaired fasting glucose: role of increase rates of gluconeogenesis. Diabetes 2007:7:1703-11.

2. Bookman ID, Pham J, Guindi M, Heathcote EJ. Distinguishing nonalcoholic statohepatitis from fatty liver: serum-free fatty acids, insulin resistance, and serum lipoproteins. Liver Int 2006;26:566-71.

3. Kawaguchi T, Ide T, Taniguchi E, et al. Clearance of HCV improves insulin resistance, beta-cell function, and hepatic expression of insulin receptor substrate 1 and 2. Am J Gastroenterol 2007;102:570-6.

4. Nannipieri M, Gonzales C, Baldi S, et al. Liver enzymes, the metabolic syndrome, and incident diabetes: the Mexico City diabetes study. Diabetes Care 2005;28:1757-62.

5. Hanley AJ, Williams K, Festa A, et al. Elevations in markers of liver injury and risk of type 2 diabetes: the insulin resistance atherosclerosis study. Diabetes 2004;53:2623-32.
6. Doi Y, Kubo M, Yonemoto K, et al. Liver enzymes as a predictor for incident diabetes in a Japanese population: the Hisayama study. Obesity 2007;15:1841-50.

7. Monami M, Bardini G, Lamanna C, et al. Liver enzymes and risk of diabetes and cardiovascular disease: results of the Firenze Bagno a Ripoli (FIBAR) study. Metabolism 2008;57:387-92.

8. Wannamethee SG, Shaper AG, Lennon L, Whincup PH. Hepatic enzymes, the metabolic syndrome, and the risk of type 2 diabetes in older men. Diabetes Care 2005;28:2913-8.

9. Haffner SM. Relationship of metabolic risk factors and development of cardiovascular disease and diabetes. Obesity 2006;14 Suppl 3:121S-7S.

10. World Health Organization. Report of a World Health Organization and International Diabetes Federation meeting: screening for type 2 diabetes. Geneva: World Health Organization; 2003.

11. Centers for Disease Control and Prevention. Prevalence of diabetes and impaired fasting glucose in adults-United States, 1999-2000. MMWR Morb Mortal Wkly Rep 2003;52:833-7.

12. Koopman RJ, Mainous AG III, Liszka HA, et al. Evidence of nephropathy and peripheral neuropathy in US adults with undiagnosed diabetes. Ann Fam Med 2006;4:427-32.

13. Glümer C, Carstensen B, Sandbæk A, Lauritzen T, Jørgensen T, Borch-Johnsen K. A Danish diabetes risk score for targeted screening: the Inter99 study. Diabetes Care 2004;27:727-33.

14. Franciosi M, De Berardis G, Rossi MCE, et al. Use of the diabetes risk score for opportunistic screening of undiagnosed diabetes and impaired glucose tolerance. Diabetes Care 2005;28:1187-94.

15. Thomas C, Hyppönen E, Power C. Type 2 diabetes mellitus in midlife estimated from the Cambridge Risk Score and body mass index. Arch Intern Med 2006;166:682-8.

16. American Diabetes Association. Standards of medical care in diabetes, 2007. Diabetes Care 2007;30: S4-S41.

17. American Diabetes Association. Screening for type 2 diabetes. Diabetes Care 2004;27:S11-S14.

18. US Preventive Services Task Force. Screening for type 2 diabetes mellitus in adults: U.S. Preventive Services Task Force recommendation statement. Rockville (MD): Agency for Healthcare Research and Quality; 2008 June. Publication No. 08-05116EF-2. Available from http://www.ahrq.gov/clinic/ uspstf08/type2/type2rs.htm. Accessed 2 September 2008.

19. Diabetes UK. Position Statement: early identification of people with type 2 diabetes. London: Diabetes UK; 2006.

20. National Center for Health Statistics, Centers for Disease Control. National Health and Nutrition Examination Survey. Data Sets and Related Documentation. 
Available from http://www.cdc.gov/nchs/about/major/ nhanes/datalink.htm. Accessed December 2007.

21. National Institute for Alcohol Abuse and Alcoholism. Clinicians Guide 2005. Available from http:// pubs.niaaa.nih.gov/publications/Practitioner/ CliniciansGuide2005/clinicians_guide.htm. Accessed 12 December 2007.

22. Pearson TA, Mensah GA, Alexander RW, et al.
AHA/CDC Scientific Statement. Markers of inflammation and cardiovascular disease. Circulation 2003; 107:499-511.

23. Sudman S, Warnecke R, Johnson T, O'Rourke D, Davis AM. Cognitive aspects of reporting cancer prevention examination and tests. Vital Health Stat, series 6, number 7. Washington DC: National Center for Health Statistics; 1994. 